Usages du français et pratiques d'enseignement en Europe balkanique, centrale et orientale - Grèce,

Serbie, Bulgarie, Moldavie, Hongrie, Allemagne, Russie - XVIIIe - XXe siècles

\title{
Conseils pour apprendre le français: une lettre d'un précepteur français (Londres, 1717)
}

\section{Vladislav Rjéoutski}

\section{(2) OpenEdition Journals}

\section{Édition électronique}

URL : https://journals.openedition.org/dhfles/3915

DOI : 10.4000/dhfles.3915

ISSN : 2221-4038

Éditeur

Société Internationale pour l'Histoire du Français Langue Étrangère ou Seconde

Édition imprimée

Date de publication : 1 juin 2015

Pagination : $9-22$

ISSN : $0992-7654$

Référence électronique

Vladislav Rjéoutski, « Conseils pour apprendre le français: une lettre d'un précepteur français (Londres, 1717) », Documents pour l'histoire du français langue étrangère ou seconde [En ligne], 54 | 2015, mis en ligne le 01 janvier 2018, consulté le 27 mars 2023. URL : http://journals.openedition.org/dhfles/3915 DOI : https://doi.org/10.4000/dhfles.3915

Ce document a été généré automatiquement le 27 mars 2023.

Tous droits réservés 


\title{
Conseils pour apprendre le français: une lettre d'un précepteur français (Londres, 1717)
}

\author{
Vladislav Rjéoutski
}

1 Le début du XviII ${ }^{e}$ siècle est marqué en Russie par des recherches et des hésitations linguistiques. Sous le règne de Pierre le Grand, la Russie s'ouvre résolument à l'Europe, mais cette ouverture est conditionnée par la maîtrise des langues étrangères. Les contacts avec différents pays d'Europe, tels que les Pays-Bas, l'Angleterre, les pays allemands, et enfin la France, poussent les Russes à apprendre des éléments des langues de ces pays. Au XVII ${ }^{\mathrm{e}}$ siècle, les Russes, même appartenant à l'élite, apprenaient rarement les langues étrangères. Il s'agit maintenant de rattraper le temps perdu pour être opérationnels dans différents domaines et activités où la connaissance des langues européennes est de rigueur: commerce, diplomatie, recrutement de spécialistes... Pierre $\mathrm{I}^{\text {er }}$ lui-même est confronté à des problèmes linguistiques lors de ses voyages en Occident et comprend donc l'importance de la maîtrise des langues européennes pour le succès de ses réformes.

2 La France reste longtemps exclue du nombre des pays avec lesquels la Russie entretient un contact direct et intensif et ce n'est qu'en 1717 que le tsar se rend finalement dans ce pays. Cependant, dès avant ce voyage le tsar fait apprendre le français à ses enfants et à ses nièces ce qui témoigne de la compréhension du rôle de cette langue dans l'éducation des princes et des aristocrates en Europe. Plusieurs de ses proches collaborateurs lui emboîtent le pas, d'autant plus que la connaissance du français est nécessitée par des considérations pratiques: les livres français commencent à être diffusés en Russie et la production livresque française est incontournable dans certains domaines tels que le génie civil et militaire par exemple; le français commence également à prendre le dessus sur le latin dans le domaine de la diplomatie.

Cependant, il est alors relativement difficile de trouver des professeurs de français en Russie: la communauté francophone est très limitée et comprend surtout des spécialistes recrutés en Europe pour réaliser des missions précises en Russie, ils ne 
peuvent donc pas remplir en même temps les fonctions d'enseignants de français. Le tsar envoie en Occident à plusieurs reprises des groupes de jeunes gens pour se former à des métiers qui n'étaient pas encore pratiqués en Russie. Plusieurs Russes profitent donc de ces séjours souvent prolongés pour apprendre les langues étrangères, y compris le français. Cet article analyse justement le cas de figure d'un jeune aristocrate russe envoyé en Angleterre pour y faire l'apprentissage du français.

4 Le «Recueil de lettres françoises $»^{1}$ qui a attiré notre attention à la Bibliothèque nationale de Russie, à Saint-Pétersbourg, est écrit d'un bout à l'autre par une seule et même main. Une lecture plus attentive montre qu'il s'agit de lettres rédigées et recopiées par un homme se trouvant à Londres vers 1717. Cet homme apprenait le français en s'entraînant à écrire des lettres. La première lettre recopiée est celle d'un précepteur résidant à Londres² .

5 Nous présenterons d'abord le parcours de l'auteur de ce recueil pour mieux comprendre le contexte, dans lequel s'inscrit cette histoire. Nous verrons ce que Londres pouvait offrir à cette époque à un apprenant de français et, plus concrètement, dans quel cadre cet apprentissage avait lieu. Finalement, en puisant dans les lettres contenues dans ce recueil, nous montrerons ce qu'on apprend sur les méthodes utilisées lors de ces cours.

\section{Un aristocrate russe à Londres}

6 Qui était l'auteur de ce recueil ? C'était de toute évidence un Russe : il mentionne sa correspondance avec des parents à Moscou et plusieurs de ses lettres tournent autour de sujets « russes » (par exemple une nouvelle visite de Pierre I ${ }^{\text {er }}$ en Europe occidentale, en 1717). En avançant dans la lecture, nous arrivons enfin à identifier ce personnage mystérieux : son nom est mentionné dans quelques lettres recopiées par lui : elles lui étaient destinées. Après avoir croisé les faits connus sur la vie du propriétaire de ce recueil avec la biographie du prince Ivan Chtcherbatov nous pouvons écarter tout doute : il s'agit bel et bien de la même personne.

7 Ivan Andreïevitch Chtcherbatov (1696-1761) est le fils du prince Andreï Dmitrievitch Chtcherbatov, seigneur russe proche du tsar Alexis Mikhailovitch, père de Pierre $\mathrm{I}^{\mathrm{er}}$. Ivan fait partie des jeunes nobles envoyés par le tsar en Angleterre pour apprendre l'art de la marine. Arrivé à Londres, probablement en $1716^{3}$, le prince y reste jusqu'en septembre 1721. Au moment où nous le trouvons, il y vit grâce au revenu de ses domaines sans aucune aide des autorités russes. Il s'initie au français, ainsi qu'à plusieurs « sciences ", en prenant des leçons auprès de professeurs privés. Pour l'heure, il n'y a aucune mention d'un apprentissage pratique de la marine; plus tard, le prince adressera au roi d'Angleterre une demande pour être admis dans la flotte britannique en qualité de volontaire, mais cette démarche n'aboutit pas car l'admission des étrangers dans la marine anglaise est alors interdite; Chtcherbatov s'adresse alors à la France pour y trouver une place similaire, sans davantage de succès.

Chtcherbatov profite de son séjour pour apprendre des langues mais également pour se renseigner sur les idées occidentales dans différents domaines, notamment en économie. Ainsi, en 1720, il traduit en russe les Considérations sur le numéraire et le commerce ${ }^{4}$ l'un des principaux écrits économiques de John Law. Chtcherbatov y ajoute sa propre introduction, dans laquelle il loue l'économiste écossais; il accompagne sa traduction de commentaires pour expliquer au lecteur russe le sens des poids et 
mesures en Angleterre et en France sans oublier de présenter les institutions britanniques et françaises. Il expose de même les principales raisons du développement du commerce en France et de l'accroissement des recettes de l'État : ce sont selon lui les banques et les compagnies de commerce. Sa traduction est envoyée à Saint-Pétersbourg en mars 1720. Le 12 juin de la même année, Chtcherbatov adresse au tsar une longue épître dans laquelle il fait plusieurs propositions ${ }^{5}$ : introduire en Russie des banques et des billets de banque et la fondation de compagnies de commerce. Il donne beaucoup de précisions sur l'organisation des banques et des compagnies commerciales et sur l'utilité et la manière d'introduire des billets de banque. Or en 1720, les initiatives de John Law connaissent un échec cuisant en France où les billets de banque se dévaluent à toute vitesse. Pierre le Grand est au courant de cette crise, mais donne pourtant l'ordre de proposer à Law des conditions très avantageuses pour l'inviter à SaintPétersbourg.

Rentré en Russie en septembre 1721, Chtcherbatov entre aux Affaires étrangères. En 1722, il est promu au rang de conseiller de commerce. L'année suivante, il est envoyé à Cadix pour préparer l'arrivée d'un consul russe. Il y écrit une «Introduction sur le commerce en Russie " dans laquelle il fait une synthèse sur le commerce russe en utilisant beaucoup de sources occidentales (Troïtski 1970). C'est sans doute lui qui écrit, à Madrid en 1725, en français, des «Observations sur le commerce des pays qui sont voisins à l'empire de Russie » (Bibliothèque nationale de Russie, Mss, Fr. Q. II. n ${ }^{\circ} 100$, 1+19 f.). Il devient ministre plénipotentiaire de Russie à Madrid en avril 1726. Dans ses nombreuses dépêches, il attire l'attention sur l'état de l'armée et de la marine espagnoles, l'économie en Espagne, ses colonies, etc. Sa carrière prend un bon départ : il est ministre russe à Constantinople en 1731, devient vice-président du collège (ministère) de Commerce en 1733, président du collège de Justice (autrement dit ministre de la Justice) en 1734 avant de revenir en Angleterre en 1739 en qualité de ministre plénipotentiaire de Russie. Peu avant ce nouveau séjour, il épouse la sœur de la comtesse Ostermann et devient ainsi parent du comte Heinrich Ostermann, l'un des plus hauts fonctionnaires russes qui dirige de fait la politique intérieure et extérieure de l'empire de Russie jusqu'à l'avènement d'Élisabeth. En 1741, une nouvelle promotion l'attend: Chtcherbatov devient conseiller privé. Il représente la cour russe à Londres, avec une courte interruption, jusqu'en 1746. C'est l'un des diplomates russes les mieux vus à la cour britannique. Rentré en Russie, il devient sénateur en 1748 (Cross 1980 : 12-13; Ivan Andreevitch Chtcherbatov).

\section{Apprendre le français à Londres : mode d'emploi}

Les lettres de Chtcherbatov nous renseignent sur le séjour à Londres de ce jeune aristocrate russe passionné par les langues et amoureux du français. Chtcherbatov mène dans la capitale anglaise une vie parfois nonchalante, parfois studieuse : il se lève souvent tard, se promène beaucoup en compagnie d'amis (sont mentionnés Messieurs Hampson et Greems, sans doute des Britanniques), passe les soirées au café «Chocolat de la Meuse ", sans doute un "haut lieu » de la diaspora française à Londres, ou au "Café de Georges ». Son mode de vie est tellement différent des passe-temps habituels d'un boyard russe - il joue maintenant au billard, boit du café, dîne en compagnie des femmes; cette existence occidentalisée va de pair avec son apprentissage des langues occidentales. 
11 Au début du XVIII ${ }^{\mathrm{e}}$ siècle, Londres est un bon endroit pour apprendre le français qui jouit d'une grande popularité dans la société anglaise: les élites britanniques maitrisent souvent bien le français, la ville est pleine d'émigrés de France, en grande partie des huguenots, dont beaucoup se consacrent à l'enseignement de leur langue. $\mathrm{Si}$, de 1480 à la fin du XVII ${ }^{e}$ siècle, environ 160 grammaires et dictionnaires de français voient le jour en Angleterre, ce nombre sera presque triplé au XVIII ${ }^{\mathrm{e}}$ siècle (Caravolas 2013 : 92).

Beaucoup des 126 lettres contenues dans ce recueil rédigé par Chtcherbatov sont des exercices de langue et de style du jeune prince. Toutes ces lettres sont marquées par un «A » ce qui permet de les distinguer de la correspondance réelle de Chtcherbatov. Les lettres sont soigneusement recopiées par le prince comme des documents sacrés témoignant de sa capacité d'écrire dans la langue de Molière. Dans plusieurs lettres, on voit une autre écriture, sans doute celle de son professeur. L'ensemble de ces documents permet de se faire une idée de la manière d'enseigner de ce professeur, que nous analyserons plus loin.

13 L'élève est convaincu de l'utilité du français. Dans une lettre - qui n'est pas marquée par un «A », mais qui a aussi été corrigée par le précepteur, il raconte sans doute une expérience personnelle ${ }^{6}$ :

Je passay hier l'après diné à parler françois avec monsieur G.: mais j'eus de la peine de le bien entendre, car la langue françoise ne luy est pas tout à fait naturelle. Aussitôt qu'il m'eut fait paroitre quelque inclination de se remettre à étudier [inséré par une autre main : $\left.\mathrm{le}^{7}\right]$ françois je ne manquay pas de vous recommander à luy. Peut étre que j'aurai la commodité de luy parler plus amplement de l'utilité de la Langue françoise [...]. (BNR, Mss, Erm., fr., $n^{\circ} 105, f^{\circ} 102$ )

Le thème de plusieurs de ces lettres est suggéré par des circonstances réelles. Une fois, Chtcherbatov demande de l'excuser d'avance pour un possible retard et explique ses raisons :

J'ose me flatter que vous ne recevrez point de mauvais œil le billet que je prens ici la liberté de vous écrire; mais qu'au contraire vous voudrez bien daigner le lire avec la même disposition de bonté dont il vous plaît toujours user envers moy. Ce qui en fait le sujet est une grace que j'ai à vous demander. Je suis demain matin engagé à aller quelque part pour une affaire pecuniaire, et s'il arrivoit que contre mon attente je fusse trop-long-temps détenu, je vous conjure de ne pas trouver mauvais, que je ne me rende pas chez vous à l'heure marquée. (BNR, Mss, Erm., fr., $\mathrm{n}^{\circ} 105, \mathrm{f}^{\circ} 111 \mathrm{v}-112$ )

Dans une autre lettre, le prince demande pardon à son professeur pour s'être trouvé au lit quand celui-ci est arrivé pour lui donner son cours. Mais il y a aussi des mots badins qui semblent être de purs exercices de style :

[A] Monsieur,

Je suis ici à croquer le marmot. Il n'y a ame qui vive dans le caffé avec qui je puisse caqueter un peu pour chasser l'ennuy. Il fait un temps si vain et si lache que j'en suis tout accablé. La chaleur qu'il fait trousse bien des gens, à ce qu'on dit. Il est mort ce matin un Agioteur françois de nation qui s'étoit morfondu à tracasser pour accumuler du bien dont il n'avoit que faire car il en regorgeoit. Les Agioteurs sont d'étranges sortes de gens. Rien ne peut assouvir leurs desirs [continué par une autre écriture $^{8}$ ] d'entasser richesses sur richesses. etc. (BNR, Mss, Erm., fr., $\mathrm{n}^{\circ} 105, \mathrm{f}^{\circ}$ 105)

Chtcherbatov semble apprécier son professeur. Cependant, pour les corrections, celuici se limite au strict minimum en faisant attention plus au style qu'à la grammaire qui 
reste assez négligée. Les enseignants de français à Londres sont nombreux à cette époque et beaucoup remplissent leurs fonctions vite et peu consciencieusement. Leur image est souvent celle de fainéants qui se font payer cher pour un travail médiocre (Caravolas $2013: 98$ ).

\section{Méthodes d'un enseignant de français à Londres}

Nous ne pouvons pas être absolument sûrs que la lettre placée au début du recueil a été écrite par l'enseignant du jeune Chtcherbatov qui a corrigé par la suite ses lettres. Cependant, certains indices nous laissent penser qu'il s'agit de la même personne: d'abord la place accordée à cette lettre dans le recueil, puis l'insistance dans cette lettre sur la lecture des œuvres de Molière qui, comme nous le verrons, occupe en effet beaucoup de place dans l'apprentissage du français par le jeune prince.

L'élève décrit ce procédé :

En cas que La Gazette de Hollande soit arrivée, je prendrai le plaisir de la lire, et tout ce que j'y trouverai de remarquable je vous le dirai. J'espère que vous voudrez bien raisonner avec moy sur les nouvelles, comme vous étes accoutumé de le faire avec tout le monde et principalement avec vos écoliers du nombre de quel je suis. (BNR, Mss, Erm., fr., $\mathrm{n}^{\circ} 105, \mathrm{f}^{\circ}$ 108)

Le prince mentionne aussi la lecture des pièces et notamment de celles de Molière qui figurent dans la liste des lectures conseillées par le précepteur :

Si vous vous trouvez demain sur le midy chez vous, je me donnerai l'honneur de vous venir rendre mes devoirs et de vous sacrifier une bonne grosse heure à la Lecture de notre Comedie. Aprés l'avoir finie nous pourrons voir le Bourgeois Gentil-homme de Moliere, si le cœur vous en dit. Je vous suis caution que vous y trouverez vôtre conte, et que vous tomberez d'accord que le ridicule des Personnages y est fort bien touché. Moliere étoit excellent homme à représenter les caracteres, et à divertir les auditeurs. Que je souhaiterois sçavoir par coeur une partie des plus beaux endroits. [ajouté par une autre main ${ }^{9}$ ] de ses comédies! (BNR, Mss, Erm., fr., $n^{\circ} 105, f^{\circ} 101 v$ )

Ce mot tombait apparemment à point nommé car la patience du professeur était passablement éprouvée si l'on en juge d'après cette phrase ajoutée par lui à l'intention de son élève : "on lui demande son assistance pour mener une vie moins dissipée " (BNR, Mss, Erm., fr., $\mathrm{n}^{\circ} 105, \mathrm{f}^{\circ} 101 \mathrm{v}$ ).

Quels livres le professeur de Chtcherbatov conseille-t-il à son élève ? Il parle des Lettres de Bussy Rabutin et de celles de Fontenelle. On trouve dans la bibliothèque du prince Mikhail Chtcherbatov, écrivain et historien russe et beau-fils du prince Ivan, un exemplaire des Lettres de messire Roger de Rabutin, comte de Bussy (Rabutin, comte de Bussy 1698). Sur une des pages du livre on voit quelques mots écrits par une main qui ressemble beaucoup à celle du propriétaire de ce recueil: «Ce livre et a Monsieur le Pri[n]ce Scherbam ». L'inscription remonte sans doute à la période où Ivan Chtcherbatov faisait ses premiers pas dans l'apprentissage du français. Les Lettres de Rabutin étaient bien connues et utilisées à des fins didactiques à travers l'Europe et jusqu'en Russie: dans les années 1730, les étudiants au collège de l'Académie des Sciences de Saint-Pétersbourg apprennent le français en traduisant ces Lettres (Mézin, Rjéoutski 2011 : vol. $2: 731$ ). 

expressions nécessaires pour mener une correspondance particulière. Dans la lettre «Au comte de Bussy Rabutin » nous lisons : « Permettez-moi, Monsieur, s'il vous plaît, qu'en vous donnant de nouvelles assurances de mes très humbles respects, je vous donne en même tems quelques nouvelles de ce qui se passe sur cette côte" (Rabutin, comte de Bussy 1698 : 9); et puis : «Voila toutes les nouvelles de ce Païs ci que j'ai crû que vous seriez bien aise de sçavoir; et je prendrai occasion de vous dire que je suis, avec un très-respectueux attachemens etc.» (Rabutin, comte de Bussy 1698: 12). Fontenelle était aussi l'un des auteurs favoris des maitres de français. On lui doit des lettres au cardinal de Fleury, à Newton, à Crousaz, etc. Mais le professeur de Chtcherbatov pense peut-être à ses lettres galantes publiées sous le nom de chevalier d'Her et rééditées de nombreuses fois (Fontenelle 1683).

Cette liste de lectures utiles pour l'apprentissage du français pourrait évidemment être complétée. Les Aventures de Télémaque de Fénelon deviennent l'un des fondements de l'enseignement de la langue française partout en Europe, pour leur message pédagogique et pour leur utilité didactique (Minerva 2003). En Angleterre, Abel Boyer, auteur de grammaires françaises et d'un dictionnaire franco-anglais, lit le Télémaque et les pièces de Molière avec ses élèves (Caravolas 2013 : 100). Les Aventures de Télémaque sont aussi connues en Russie (Drews 1992). À peu près dans les mêmes années, le gouverneur français Pirard dicte à son élève russe, le jeune prince Tcherkasski, des morceaux tirés des " Maximes pour se conduire sagement dans le monde » attribuées à Fénelon (Archives d'État des actes anciens à Moscou, infra - RGADA, fonds 11, d. 291, fo $25-25 v)^{10}$. Bossuet se prête aussi fort bien à des dictées, par exemple son Discours sur l'histoire universelle dont le début est devenu célèbre : "Quand l'histoire seroit inutile aux autres hommes, il faudroit la faire lire aux princes ${ }^{11}$.

La lecture de Rabutin et de Fontenelle, la composition de lettres ont porté leurs fruits : le jeune Chtcherbatov écrit, certes, non sans fautes, mais d'une plume alerte en se servant d'une multitude d'expressions toutes faites: "J'ay eu l'honneur de recevoir vôtre lettre ", «Je suis bien faché de [...] ", «Je prens la liberté de vous prier, de vouloir bien vous donner la peine $[. .]$.$» , « je me sers de cette occasion pour vous asseurer avec$ combien d'attachement je suis [...]», «Passez moy quelque negligence, je vous en prie, et ayez la bonté de [...] », « je finis par des protestations d'attachement et de respect », "Je vous conjure de croire que j'ay l'honneur d'etre veritablement [...]», «Je ne manquerai pas de vous faire savoir [...] », etc., etc.

Enfin, le professeur le dissuade de faire des traductions de l'anglais en français ce que Chtcherbatov avait, semble-t-il, l'habitude de faire. Mais ce n'est pas pour le détourner complètement des traductions car, à cette époque, c'est l'une des méthodes couramment utilisées dans l'apprentissage des langues. Le précepteur conseille de prendre comme base un texte français, de le traduire en anglais, et de le retraduire en français en comparant le résultat au texte de départ. Chtcherbatov aime traduire : on trouve dans le même carnet plusieurs traductions faites surtout du français et de l'anglais vers le russe (BNR, Mss, Erm., fr., $\mathrm{n}^{\circ} 105$, fo 1-5, etc.).

\section{Conclusion}

Ce recueil curieux est l'un des premiers documents écrits par un Russe en français. L'histoire se passe à l'étranger et ce n'est pas tout à fait un hasard : en Russie à cette 
époque, le français n'avait pas encore acquis pleinement ses lettres de noblesse et, surtout, les enseignants de français y sont alors rares. Le prince Ivan Chtcherbatov fait donc figure de précurseur en adoptant à l'égard de la « langue de l'Europe » l'attitude passionnée d'un converti.

Cette source montre comment l'apprentissage du français pouvait se faire au moment où la Russie s'ouvrait à l'Europe. Ces lettres permettent aussi de découvrir les procédés utilisés dans l'enseignement du français à Londres à cette époque. Parmi ceux-là, l'écriture de lettres occupe évidemment une place particulière si l'on pense au parcours ultérieur de ce Russe. La suite de la carrière du prince Chtcherbatov montre en effet à quel point la maîtrise du français et notamment de l'art d'écrire des lettres en français ont été cruciaux pour lui : la connaissance des théories économiques de John Law, la traduction, le travail en qualité de diplomate en Espagne et plus tard en Angleterre, tout cela était conditionné par la maîtrise des langues étrangères. La correspondance diplomatique de Chtcherbatov du temps de sa présence en Espagne montre qu'il échangeait en effet avec plusieurs diplomates européens et que le français lui servait de langue de communication en cette occasion. Il l'utilise aussi, bien qu'occasionnellement, avec des diplomates russes, alors qu'à cette époque les Russes, même exerçant le métier de diplomate, font leurs échanges encore normalement en russe (BNR, Mss, Erm, $\mathrm{n}^{\circ} 76$ ). Il anticipe donc sur l'usage du français comme langue de communication entre les Russes, qui ne sera véritablement adopté que sous le règne de Catherine II (1762-1796). Londres étant, au début $\mathrm{du}$ XVIII ${ }^{\mathrm{e}}$ siècle, une capitale cosmopolite où le français jouait un rôle non négligeable, la période de son apprentissage a donc été très formatrice à différents égards : la manière de vivre, de s'habiller, de manger, de communiquer, tout cela a été de grande importance pour ce jeune Russe, mais son capital culturel le plus précieux était sans doute sa maîtrise des langues et du français d'abord et surtout.

\section{Annexe}

En annexe nous publions la première lettre de ce recueil, écrite par le précepteur du prince Chtcherbatov. On verra que l'orthographe et même la grammaire de cette lettre sont loin d'être parfaites. Il ne faut pas oublier qu'il s'agit d'une copie faite par le jeune Chtcherbatov pour son propre usage. C'est peut-être à l'élève que ces erreurs sont imputables, si ce n'est pas à son précepteur...

Source : Bibliothèque nationale de Russie, Mss, Erm., fr., $\mathrm{n}^{\circ} 105$, «Recueil de pièces curieuses fait depuis le 26 Octobre 1717. En français, en anglais et en russe». Le texte est publié en respectant l'orthographe de l'original.

[f॰ $113 v$ ]

Monsieur,

Quelques instance que j'aye pû faire aupres de mes ecoliers, pour les porter à changer le temps de nos exercices, il ne m'a pas été possible de réüssir. J'en suis au desespoir, puisque j'y pers l'honneur d'étre employé par vous Monsieur. Agréez pourtant, je vous prie que je vous sçache tres-bon gré de la bonne volonté, que vous avez pour moy, et que j'en conserve du souvenir. Puisque vous avez une si fort ardeur pour nôtre Langue, permettez moy dire ma pensée sur les moyens de vous la rendre familiere. Il n'y a pour cela qu'à lire beaucoup, à écrire beaucoup, et à parler beaucoup. Prenez s'il vous plait, 
la peine de vous attacher sur tout à la lecture des comedies de Moliere, des Lettres de Bussy, de celles de Fontenelles, et aux Gazettes de Paris, et d'Amsterdam, qui sont les mieux écrites. Il est d'une absolüe necessité de faire un recueil [ [ $\left.\mathrm{f}^{\circ} 113\right]$ de termes choisis, d'expressions élegantes, et de tours qui sont du génie de nôtre Langue pour vous en former un stile selon la [sic! - V.R.] caractere de vôtre humeur. Tout cela doit tirer des pièces que vous lirez et vous faire faire beaucoup de questions par vôtre Maitre, sur ce que vous aurez lû. Mais de grace, ne suivez point la route battüe dans vos traductions. Je veux dire de ne point traduire l'anglois en françois. Vous apprendrez bien mieux le genie de nôtre Langue et cette liaison des termes, qu'il est si difficile d'attraper, en traduisant du françois en Anglois et en retraduisant ensuite le même Anglois en françois pour comparer ce que vous aviez fait avec l'original. On doit s'assûrer de travailler utilement en suivant cette methode, au lieu que par l'autre on ne fait rien qui vaille de long temps. Qu'un de vos principaux soins soit de vous acquerir une prononciation facile et aisée en lisant tout haut devant un Maitre attentif, et qui s'y attend. La lecture des comedies et de vers est celle, qui est la plus utile pour ce dessein. Il faudroit souvent composer des lettres, ou de petits entretiens, et vous efforcer d'y faire entrer les expressions et les termes, que vous aurez recüellis. Vous n'aurez pas fait cela dix à douze fois, que vous vous [ $\left.\mathrm{f}^{\circ} 112 v\right]$ appercevrez d'un succes qui vous fera plaisir. Je n'en dirai pas d'avantage, sur ce chapitre crainte de vous ennuyer. Je dois même vous faire mille excuses d'avoir pris tant de liberté. Mon intention a été de vous de dommager en quelque maniere du temps, que je vous fis perdre hier, car je crains fort de vous avoir arraché de quelque occupation serieuse et importante. J'ose cependant me flatter qu'elle ne vous sera pas désagreable cette intention, et que vous voudrez bien me permettre d'etre avec tout le respect, que je vous dois,

Monsieur,

Vôtre tres humble et tres ob: serviteur.

\section{BIBLIOGRAPHIE}

ANTRAIN, Abbé charuel d' (1768). La Rhétorique des savants dédiée à Monseigneur le Comte de S. Florentin contenant des pièces choisies des plus célébres poëtes et orateurs. Paris.

BOSSUET, Jacques-Bénigne (1836). CEuvres complètes de Bossuet, évêque de Meaux. T. 10. Paris.

CARAVOLAS, Jean Antoine (2013). « La contribution des précepteurs à l'avancement de la didactique des langues ». In Vladislav Rjéoutski et Alexandre Tchoudinov (dir.). Le Précepteur francophone en Europe. XVII ${ }^{e}$-XIX ${ }^{e}$ siècles. Paris : L'Harmattan, 89-113.

CROSS, Anthony G. (1980). « By the Banks of the Thames ». Russians in Eighteenth Century Britain. Newtonville, ORP, 12-13.

DREWS, Peter (1992). « Die Slawishen Abenteuer des Telemach (1715-1815) ». Zeitschrift für Slavishe Philologie, 52, 231-256. 
FÉNELON, François Salignac de la Mothe (1787). Cuuvres de M. François de Salignac de La Mothe Fénelon, précepteur des enfants de France, archevêque de Cambrai. T. 3. Paris.

FONTENELLE (1683). Lettres diverses de Mr le chevalier d'Her***. Paris : C. Blageart.

IVAN ANDREEVITCH CHTCHERBATOV - notice biographique en ligne : <http://

www.rusdiplomats.narod.ru/xerbatov-ia.html> (10.01.2015).

MÉZIN, Anne \& RJÉOUTSKI, vladislav, dir. (2011). Les Français en Russie au siècle des Lumières. 2 vol. Ferney-Voltaire : CIEDS.

MINERVA, Nadia, dir. (2003). 'Les Aventures de Télémaque'. Trois siècles d'enseignement du français. Documents pour l'histoire du français langue étrangère ou seconde, 30 \& 31.

RABUTIN, comte de BUSSY, Roger (1698). Les Lettres de messire Roger de Rabutin, comte de Bussy, Lieutenant General des Armées du Roi, et Mestre de Camp General de la Cavalerie Françoise et Etrangere. Nouvelle édition avec les Reponses, t. 3, à Paris, chez Florentin et Pierre De l'aulne. Cote de la Bibliothèque nationale de Russie : 6.58.11.10. Nous remercions Vladimir Somov et Sergueï Korolev pour leur aide.

TROITSKI, S. M. (1970). « Sistema Djona Lo i eio rousskié posledovateli ». Franko-rousskié ekonomitcheskié sviazi [S. M. Troïtski, « Le 'système' de John Law et ses adeptes russes », Les liens économiques franco-russes]. Moscou-Paris : Naouka. En ligne : <http://www.vostlit.info/> (février 2012).

\section{NOTES}

1. Contenues dans le « Recueil de pièces curieuses fait depuis le 26 Octobre 1717. En français, en anglais et en russe ", Bibliothèque nationale de Russie (infra - BNR), Mss, Erm., fr., n ${ }^{\circ} 105$. Le manuscrit commence des deux côtés du volume, la pagination est donc décroissante dans les citations ci-dessous.

2. Nous la publions en annexe.

3. Les lettres dont nous disposons, sont datées de 1717, mais il semble que Chtcherbatov était déjà à Londres bien avant.

4. Sorties à La Haye en 1705, le prince précise qu'il traduit le texte de l'anglais.

5. Le texte de cette lettre est publié dans : Troitski 1970. Le manuscrit se trouve à la Bibliothèque nationale de Russie, Mss, Erm., d. 122-a, fo 1-22v.

6. Toutes les citations qui suivent respectent l'orthographe de l'original.

7. Note de l'auteur VR.

8. Note de VR.

9. Note de VR.

10. Voir par exemple Fénelon $1787: 3: 532-533$, où ces vers sont intitulés « La Sagesse humaine ou le portrait d'un hônnête homme ». Parfois cette pièce figurait sans nom d'auteur (Antrain 1768 : 448).

11. L'une des dictées données par Pirard au prince Tcherkasski est tirée de cette œuvre : RGADA, fonds 11, d. 291, fo 40. Voir ce discours dans Bossuet 1836 : X : 151-311. 


\section{RÉSUMÉS}

En exploitant une source inédite conservée à Saint-Pétersbourg, l'auteur analyse un cas d'apprentissage du français par un aristocrate russe, à Londres au début du XVIIIe siècle. Anecdotique à première vue, cette histoire permet néanmoins de bien saisir certaines caractéristiques de l'étape initiale dans l'apprentissage des langues étrangères en Russie. Cette source permet de voir également certaines méthodes d'apprentissage du français à Londres à cette époque et le rôle de cet apprentissage pour ce jeune Russe qui allait débuter une carrière de diplomate et de fonctionnaire d'État.

INDEX

Mots-clés : apprentissage du français, aristocratie russe, méthodes d'apprentissage des langues, Londres, XVIIIe siècle.

Keywords : learning French, Russian aristocracy, methods of learning languages, London, 18th century.

\section{AUTEUR}

\section{VLADISLAV RJÉOUTSKI}

Deutsches Historisches Institut Moskau 